\title{
Heritable Cardiac Conduction and Myocardial Disease: From the Clinic to the Basic Science Laboratory and Back to the Clinic
}

\author{
Elizabeth A. Sparks ${ }^{\text {a }}$ Konstantinos Dean Boudoulas ${ }^{\mathrm{b}}$ Subha V. Raman ${ }^{\mathrm{b}}$ \\ Takeshi Sasaki $^{c}$ Harry L. Graber ${ }^{\text {b Steven D. Nelson }}{ }^{\text {b Christine E. Seidman }}{ }^{d}$ \\ Harisios Boudoulas ${ }^{b}$ e \\ a Johns Hopkins Hospital, Baltimore, Md., ${ }^{b}$ Division of Cardiovascular Medicine, Department of Internal Medicine, \\ The Ohio State University, Columbus, Ohio, and ${ }^{\mathrm{C} H a r v a r d}$ Medical School and ${ }^{\mathrm{d}}$ Cardiovascular Genetics Center, \\ Brigham and Women's Hospital, Department of Genetics, Harvard Medical School, Boston, Mass., USA; \\ eBiomedical Research Foundation, Academy of Athens, Athens, Greece
}

\section{Key Words}

Heritable cardiomyopathy $\cdot$ Conduction system disease $\cdot$ Lamin A/C

\begin{abstract}
A close collaboration between the physicians-scientists of the Division of Cardiology, The Ohio State University and the basic scientists of the Department of Genetics, Harvard Medical School was essential to define the multiple phenotypic expressions and the genetic abnormalities in the heritable conduction and myocardial disease in a family from central Ohio (Family OSU). The Family OSU presents evidence of sequential hierarchical progression through multiple cardiac phenotypes (sinus bradycardia, atrioventricular conduction defects requiring pacemaker, supraventricular arrhythmias including atrial fibrillation, heart failure, and sudden cardiac death) on a decade-to-decade basis. In this setting, each phenotype may be mistakenly considered as a specific diagnosis by physicians working without a pedigree or long-term follow-up. Genetic analysis, however, confirms lamin A/C mutation. The role of the physician-scientist and the basic scientist for the study of heritable disorders is equally important but different. Only the physician-scientist, however,
\end{abstract}

who is in constant contact with the patient understands the complexity of the disease. The physician-scientist with an interest in a particular disease can guide the basic scientist to define molecular mechanisms of that disease and by extension learn important lessons for other diseases.

Copyright $\odot 2011$ S. Karger AG, Basel

\section{Introduction}

A close collaboration between the physician-scientists of the Division of Cardiology, The Ohio State University, Columbus, Ohio, USA, and the basic scientists of the Department of Genetics, Harvard Medical School, Boston, Mass., USA, was essential to define the phenotypic expressions and the genetic abnormalities in the heritable conduction and myocardial disease of lamin A/C (LMNA) $[1,2]$.

Privately published historical details of the immigration of a young couple from Bavaria, Germany to Maryland, USA and then to central Ohio in 1830 provided the background for these studies. Published family records were supplemented by countless individual interviews, and the family pedigree was constructed beginning with

\section{KARGER}

Fax +41613061234 E-Mail karger@karger.ch www.karger.com (c) 2011 S. Karger AG, Basel

$0008-6312 / 11 / 1183-0179 \$ 38.00 / 0$

Accessible online at:

www.karger.com/crd
Prof. Harisios Boudoulas

Biomedical Research Foundation, Academy of Athens

Soranou Ephessiou Str. 4

GR-11527 Athens (Greece)

E-Mail boudoulas@bioacademy.gr 


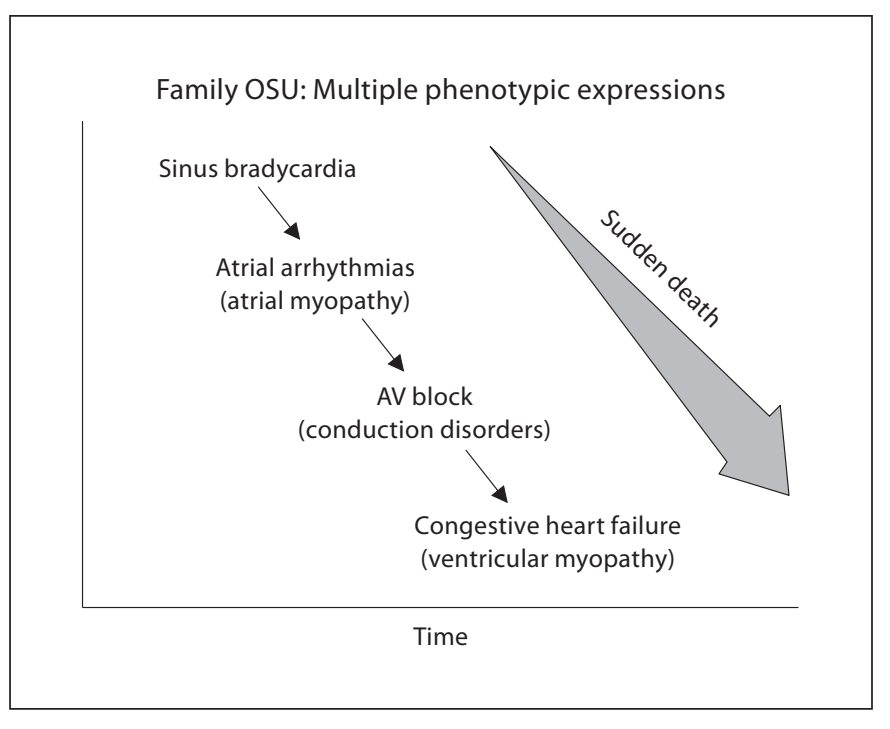

Fig. 1. The progression of age-related phenotypes in the heritable cardiac conduction and myocardial disease as seen in the Family OSU.

this immigrant couple. The father born in 1803 and the mother born in 1804 are designated in generation I. The father died at age 59 and is presumed to be the affected parent. The mother died at age 83 . Ten children were born to this union, 4 of whom were affected by the disease. The second child died and was buried at sea en route to America; this child has not been included in the pedigree. The offspring of those 4 children form the material of this study.

This family (Family OSU) has been extensively studied over the last five decades. Affected family members presented with various cardiac phenotypes at different ages and stages of the disease. It is emphasized that the definition of phenotypes in heritable diseases with multiple phenotypic expressions requires careful and longitudinal clinical observation. Close collaboration between clinical and basic scientists is essential for phenotypegenotype correlation.

\section{Definition of the Phenotype - Pedigree Construction}

\section{Clinical Presentation}

In the 1960s and 1970s, patients with high-grade atrioventricular (AV) block from central Ohio were admitted to The Ohio State University Medical Center for pacemaker implantation, an open chest procedure at the time. Careful family histories revealed shared ancestors, confirming blood relationship among patients with AV block. Furthermore, male-to-male transmission of the cardiac phenotype confirmed autosomal dominant transmission of cardiac conduction system disease. The subsequent emergence of heart failure indicated the coassociation of myocardial disease [1].

The clinical manifestations of the disease in the Family OSU typically emerged in the second and third decades of life with mild prolongation of the PR interval on the electrocardiogram associated with sinus bradycardia (resting heart rate less than 50 beats per minute). In addition, premature atrial and ventricular contractions associated with palpitations and fatigue were noted. During the fourth and fifth decades of life, patients developed persistent or permanent supraventricular arrhythmias, including atrial fibrillation, followed by high-grade AV block requiring permanent pacemaker implantation. Between 30 and 70 years of age, patients developed heart failure. Sudden cardiac death occurred between 35 and 65 years of age, but most often in the advanced stage of the disease (fig. 1) [1-4].

In the earlier retrospective studies, the phenotypic expressions of the disease at the various stages were only defined in different patients. More recently, after longterm follow-up of greater than 20 years, the entire phenotypic expression of the disease from the early stages to death was demonstrated in the same patient. Typically, the phenotypic expressions of the disease (that is, age of initial presentation, progression and death) were different in the various families; however, close relatives demonstrated a similar course of the disease as illustrated in figure 2. A 9-generation pedigree from sibling 5 is shown in figure 3 .

\section{Laboratory Findings}

High-resolution electrocardiogram prior to the development of atrial fibrillation demonstrated a decrease in P-wave amplitude and an increase in the duration of the P-wave [4]. Electrophysiologic studies in several patients demonstrated sinus node dysfunction, intra-atrial and AV node conduction abnormalities with normal conduction distal to the His [3].

Left atrial volumes obtained from the echocardiogram using the area-length method, biplane technique, demonstrated an increase in left atrial maximal volume several years prior to the development of atrial fibrillation. Thus, the data indicated that left atrial myopathy preceded the development of atrial fibrillation [4]. As a general rule, left ventricular size increased and left ventricular function decreased in patients with heart failure; 


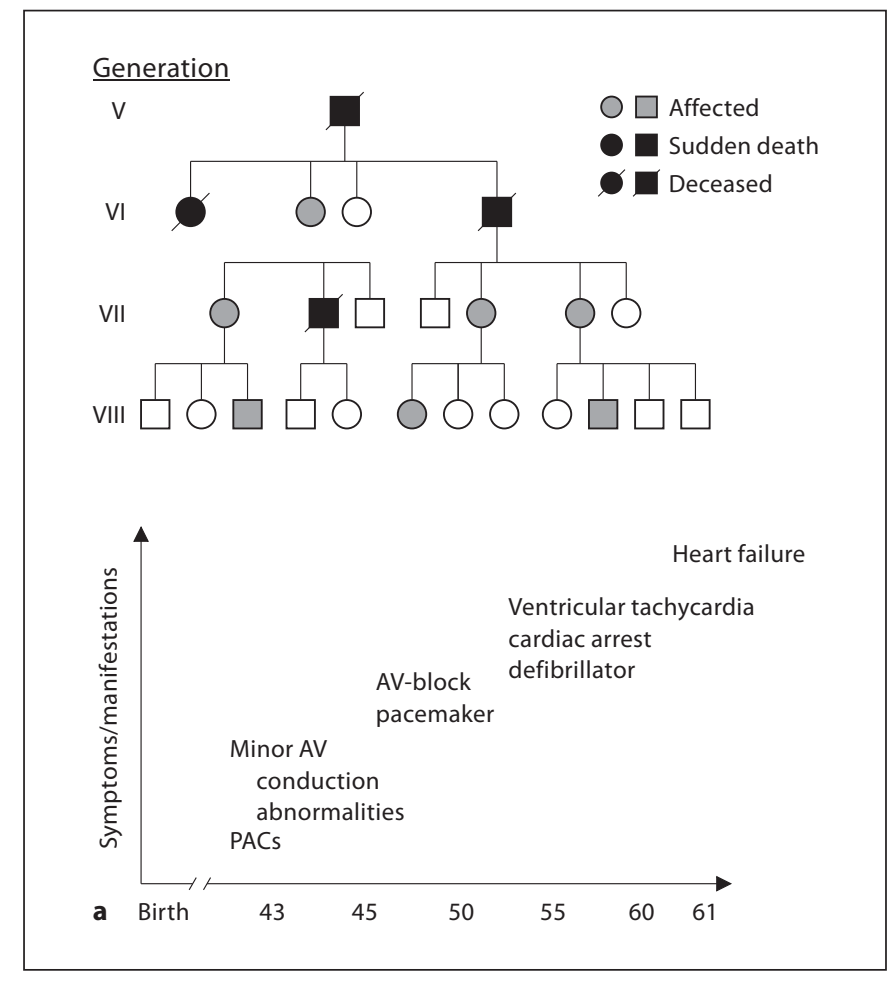

Fig. 2. a Pedigree illustrating a subset of affected Family OSU members and clustering of sudden cardiac death. The natural history of the mother is shown. The natural history of the disease in her son and daughter are illustrated in $\mathbf{b}$ and $\mathbf{c}$, respectively. $\mathrm{PAC}=$ Premature atrial contraction. Circle: female; square: male.
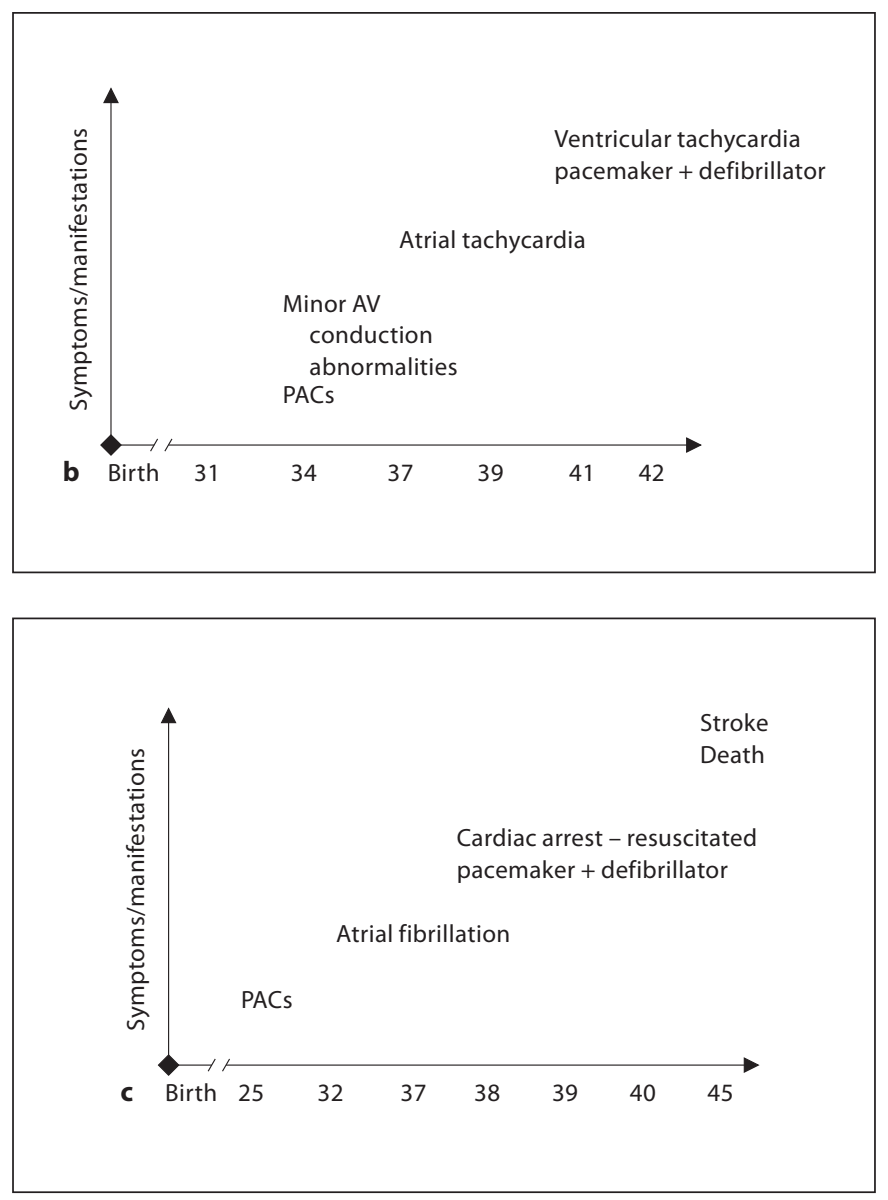

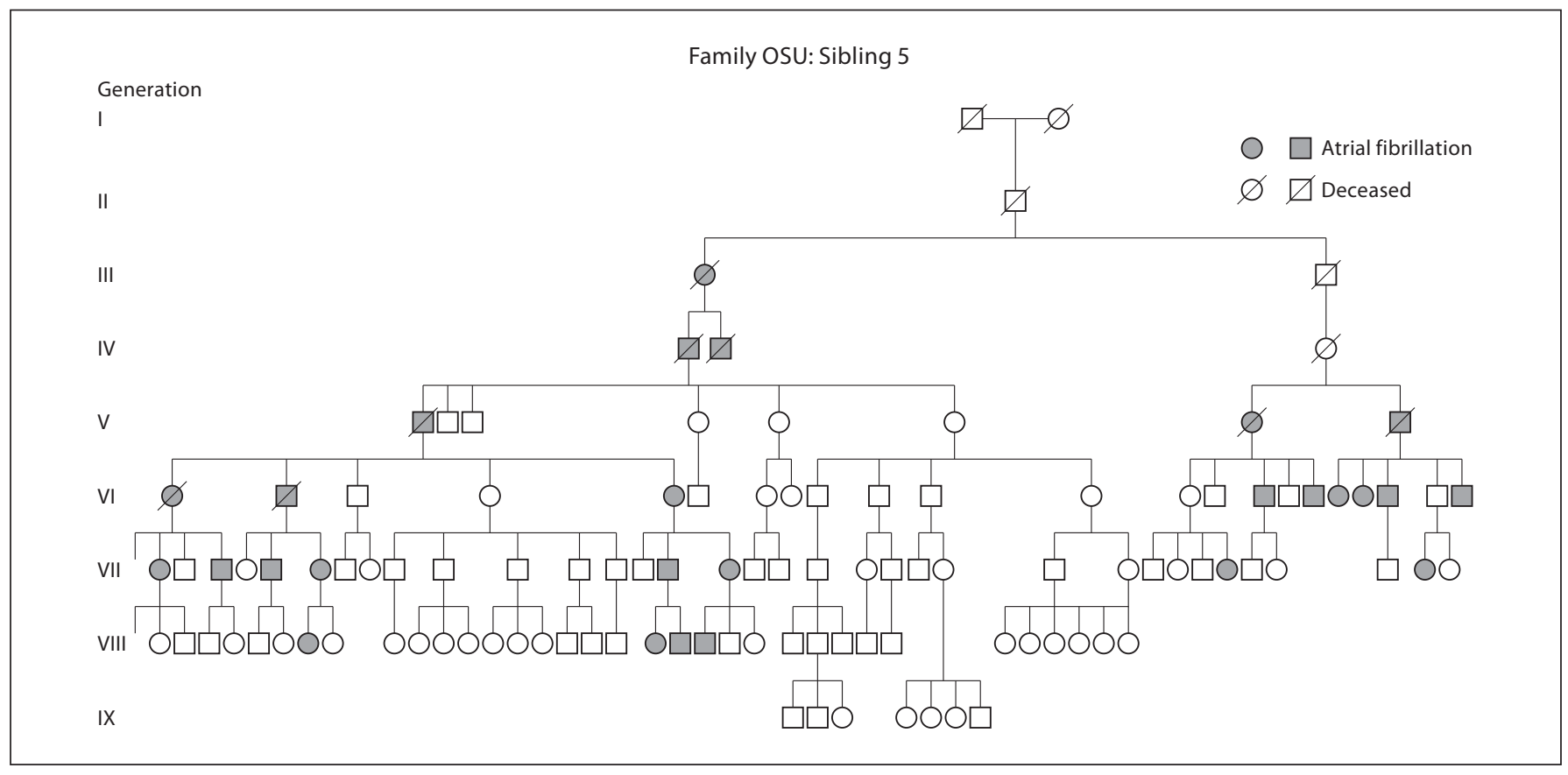

Fig. 3. Family OSU: 9-generation pedigree of sibling 5 (generation II). Circle: female; square: male. 


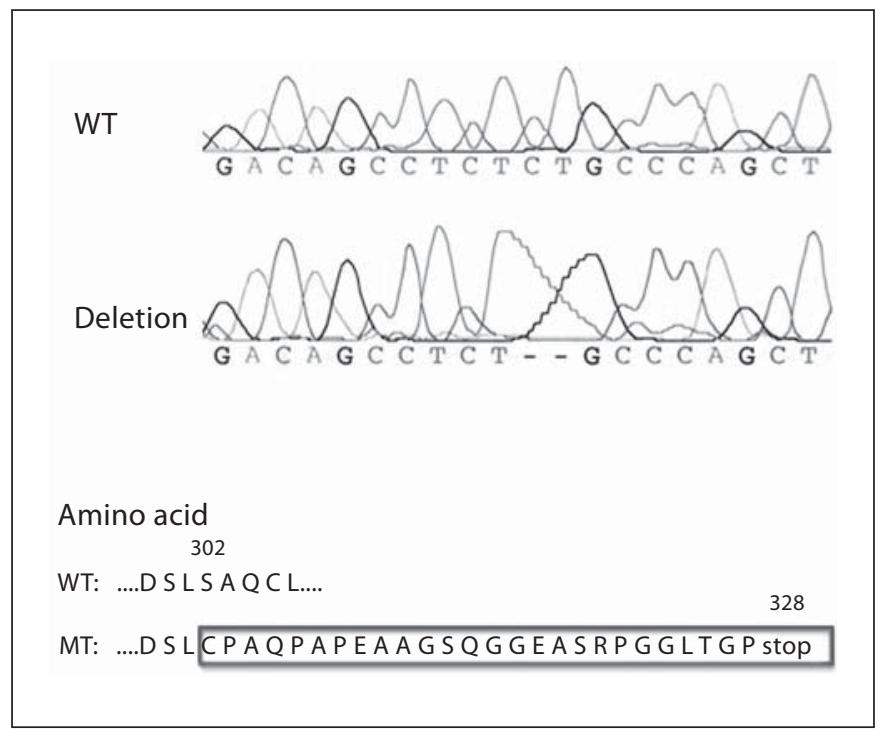

Fig. 4. Deletion of cytosine (C) and thymine (T) in the lamin gene (LMNA) are shown. The deletions result in a sequence of amino acid changes beginning at position 302 (black box) and eventually leading to the amino acid substitution of cysteine for serine at position 328 forming a premature stop codon with protein truncation. $\mathrm{WT}=$ wild type; $\mathrm{MT}=$ mutation.

however, heart failure in the presence of normal left ventricular function and dimensions was thought to be related to diastolic dysfunction.

Coronary arteriography was performed in a large number of patients who developed heart failure. As a general rule, affected individuals did not demonstrate significant obstructive coronary artery disease.

Autopsies on 8 affected individuals demonstrated marked left atrial dilatation in the majority of cases with moderate left ventricular dilatation. Further, there was severe fibrosis in the mid myocardium of the posterior left ventricular wall and basilar intraventricular septum [2]. Histologic studies performed on specimens obtained during the autopsy demonstrated moderate to severe fibrosis in the sinus node and AV node. Myocyte hypertrophy, fibrosis and degeneration ranging from mild to severe were also found; these abnormalities were more prominent in the atria compared to the ventricles $[2,5]$.

\section{From the Clinic to the Basic Science Laboratory}

The medical and family history confirmed genetic inheritance with autosomal dominant transmission and multiple phenotypic expressions (fig. 1). To define the ge- netic abnormalities of the disease, a close collaboration was initiated in the early 1990s with the Department of Genetics, Harvard Medical School in Boston, Mass. (Christine E. Seidman and Jonathan G. Seidman) [2, 6]. Family-wide genotyping was performed in more than 300 family members. DNA was isolated from $20 \mathrm{ml}$ of whole blood as described previously. A mutation in LMNA gene that encodes the lamin A/C protein was identified. The LMNA gene is located on chromosome 1q21.2 and consists of 12 exons and 3,204 nucleotides. A 2-base-pair nucleotide deletion was discovered in exon 5 of the LMNA gene at position 906 and 907, a cytosine and thymine, respectively (fig. 4). The deletions result in a sequence of amino acid changes beginning at position 302 and eventually leading to the amino acid substitution of cysteine for serine at position 328 forming a premature stop codon with protein truncation. Confirmation was performed using restriction enzyme digestion.

Lamins are intermediate filament proteins and are a major component of the nuclear lamina separating the nuclear envelope from the nuclear matrix $[6,7]$. Lamin $\mathrm{A} / \mathrm{C}$ is found in all differentiated cells and plays an important role in transcriptional regulation and maintaining the organization and structure of the inner nuclear membrane, nuclear pore complexes and heterochromatin. Mutations related to the LMNA gene result in a broad array of diseases classified as laminopathies including dilated cardiomyopathy with conduction system disease, lipodystrophy and muscular dystrophy [6-11]. The mechanisms by which LMNA mutations produce these different pathologies are incompletely understood.

The lamin A/C protein interacts with a variety of proteins including lamin B receptors, lamin-associated polypeptides and emerin, to name a few. Dysfunctional lamin A/C may result in abnormal protein-to-protein interactions causing the mislocalization of some interacting proteins producing cellular disorganization. Cardiovascular diseases related to lamin $\mathrm{A} / \mathrm{C}$ has been described in several families predominantly in late stages of the disease. Even though lamin $\mathrm{A} / \mathrm{C}$ is present in all differentiated cells, the disease in the Family OSU is expressed primarily in the heart [1-4]. To define potential significant cardiac proteins that interact with lamin A/C, an experiment using a yeast two-hybrid screen with human lamin $\mathrm{A} / \mathrm{C}$ as bait and human heart cDNA library as prey was performed. A novel protein, chromosome 6 protein (C6P), was found to interact with amino acids $14-116$ of lamin A/C [unpubl. data]. This protein is encoded on chromosome $6 \mathrm{p} 12.1$ whose sequence predicts nuclear localization [12]. C6P has been found to be mostly ex- 
Fig. 5. Cardiac pathology in MOC6P-injected zebrafish embryos decreasing the level of C6P expression. Uninjected (a) and MOC6P-injected (b) zebrafish embryos demonstrating cardiac swelling and pericardial edema (red arrow; gray arrow in the print version) consistent with heart failure at $72 \mathrm{~h}$ after fertilization. Hematoxylin and eosin staining of longitudinal sections of the heart and pericardium reveal normal cardiac morphology of uninjected (c) and pericardial effusion $\left(^{*}\right)$ in MOC6Pinjected zebrafish embryos (d).
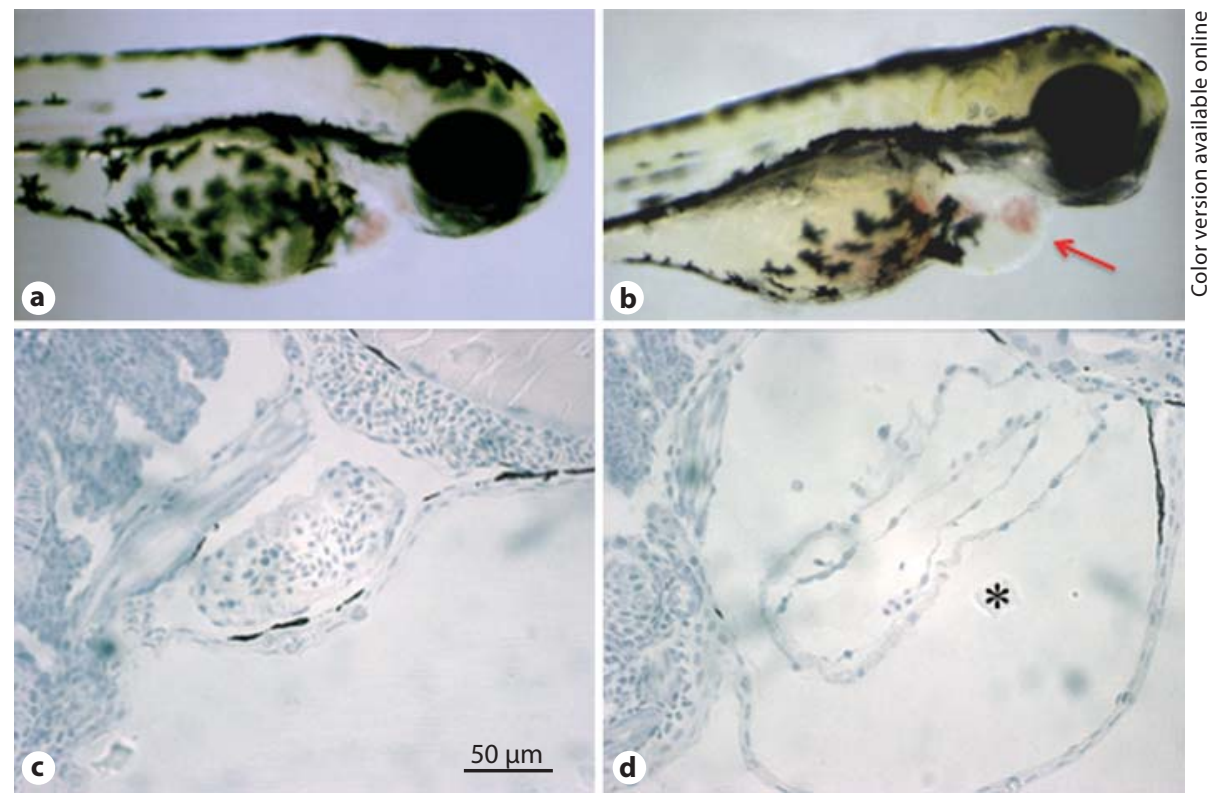

pressed in cardiac tissue in adult mouse by mRNA Northern blot analysis and in mouse and zebrafish hearts by whole mount in situ hybridization. To test whether the $\mathrm{C} 6 \mathrm{P}$ has a cardiac function, anti-sense morpholino oligonucleotide C6P (MOC6P) was injected into zebrafish embryos to decrease the level of C6P expression. MOC6P zebrafish showed cardiac swelling and pericardial edema, consistent with heart failure (fig. 5). It has been speculated that the lamin A/C mutation may result in an abnormal interaction between lamin A/C and C6P protein, thus altering the proper function of $\mathrm{C} 6 \mathrm{P}$ causing a cardiac specific phenotype of myocardial dysfunction [12]. This data provides insight to the possibility of abnormal protein-to-protein interactions as a potential mechanism on why certain laminopathies affect primarily the heart sparing other organs, although lamin $\mathrm{A} / \mathrm{C}$ is present in most tissues.

\section{From the Basic Science Laboratory to the Clinic}

Phenotypic expression of the disease combined with genetic analysis in each individual family member were important factors leading to the clinical decision for implantation of a pacemaker and/or implantable cardioverter defibrillator (ICD) [1-3]. Pacemaker therapy was initially instituted in the presence of symptomatic bradycardia or complete AV block with epicardial electrodes and fixed-rate pacemakers. More recently, pacemakers are implanted at earlier stages of the disease based on clinical history, family history and genetic analysis, including transvenous right ventricular pacing and biventricular pacemaker with or without an ICD. Right ventricular pacing, however, produces left ventricular dysynchrony and dysfunction, which may accelerate the natural course of the disease [13]. The effect of biventricular pacing in the course of this disease in genetically affected family members needs to be further evaluated.

Sudden cardiac death is a significant occurrence in the natural history of the disease. Characteristics of sudden cardiac death in this family, as reported previously, include: (1) age greater than 30 years; (2) a parent who experienced sudden death; (3) reduced functional capacity associated with left ventricular dysfunction, and (4) prior pacemaker therapy for symptomatic bradycardia or advanced AV block. Thus, ICD implantation is indicated in genotype-affected patients with high-risk features for sudden cardiac death as defined above [3]. Indeed, in several high-risk patients, ICD implantation proved to be life-saving since ventricular tachycardia/fibrillation was terminated by appropriate ICD discharge aborting sudden cardiac death. To date, the effect of ICD therapy on survival in the Family OSU has been positive, and supports the proposal for primary prevention of sudden cardiac death in patients with lamin A/C mutation. Pacemaker and ICD therapy has significantly impacted the natural history of the disease. 


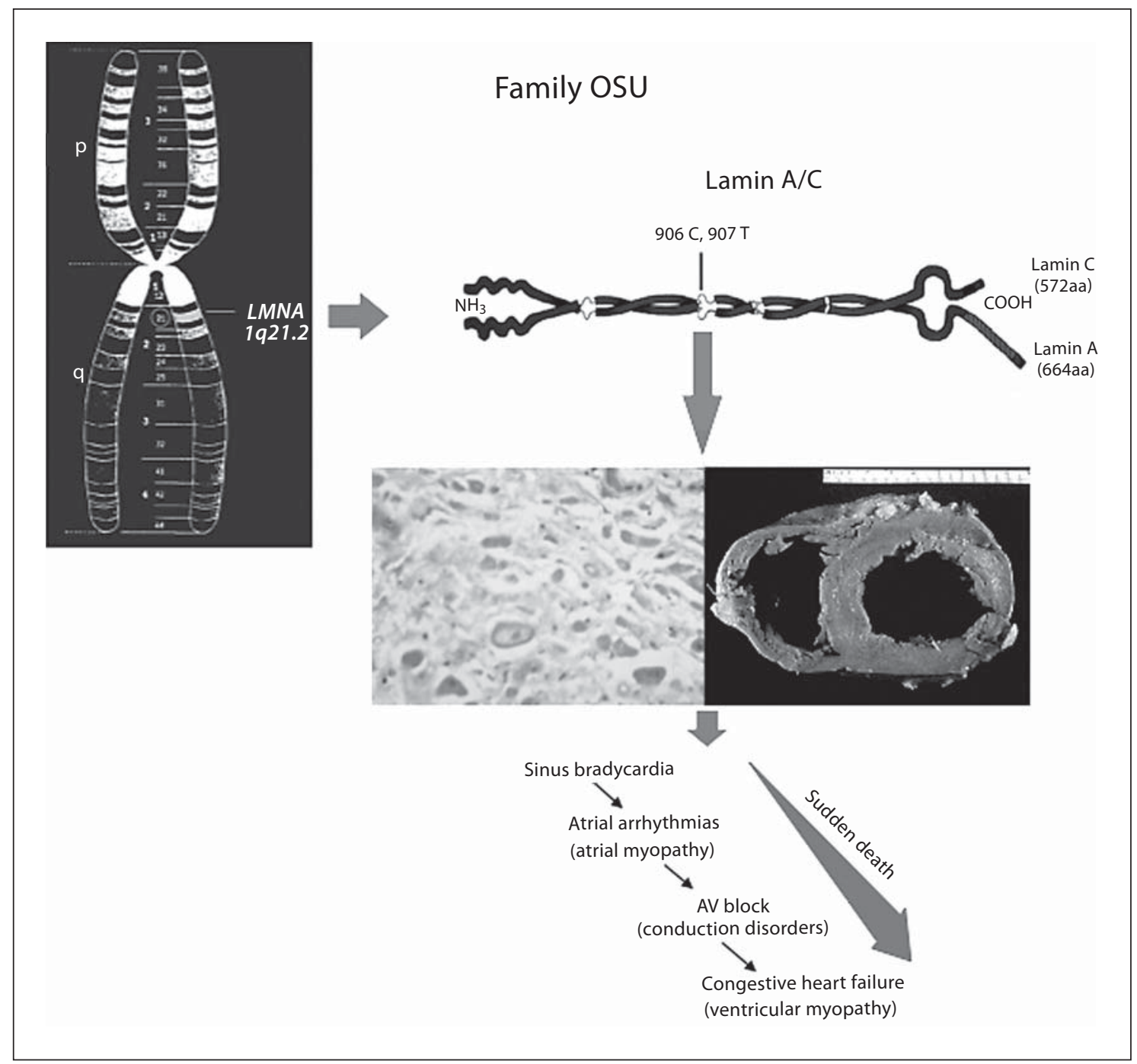

Fig. 6. Genotype-phenotype correlates in the Family OSU. A deletion of cytosine (C) and thymine (T) in the lamin gene (LMNA) results in myocardial (atrial, ventricular), sinus node and AV node fibrosis. These abnormalities are responsible for the clinical manifestations of the disease. Atrial (middle left) and ventricular septal fibrosis (middle right, from [2]) as well as the clinical manifestations of the disease are shown.

More recently, the fibrosis pattern demonstrated in the ventricular wall at autopsy has also been demonstrated on magnetic resonance imaging in genetically affected members during the early stages of the disease prior to pacemaker implantation. The presence of fibrosis corresponded to PR interval prolongation compared to fibrosis-negative patients $[14,15]$. This method may help to define early myocardial involvement in genotype-positive family members without clinical manifestations. The effect of early treatment with agents that modulate collagen synthesis and degradation can be tested in vivo in those patients [16].
All family members are potentially at risk for developing conduction system and myocardial disease. Genetic analysis will precisely define the risk of each individual for developing this inherited cardiomyopathy. Family members without the mutation have no need for longitudinal and expensive clinical evaluations; they are also free of social as well as psychological issues associated with the disease. In addition, genotyping will assist in classifying individuals with non-specific findings, such as mild sinus bradycardia, subtle PR prolongation, atrial premature contractions and the like, that are not related to the LMNA mutation, thus reassuring individuals they 
are not at risk for developing the disease (that is genotype negative). The physician can focus on genotype-positive individuals.

\section{Concluding Remarks}

The natural history and phenotypic expression of the cardiovascular disorders associated with lamin A/C mutation in the Family OSU were documented from published family records, numerous family interviews and clinical care beginning in the late 1950s. Slow heart rate, irregular heart rhythm, heart failure and sudden cardiac death are significant features of the disease. The chronology of phenotypic expression has subsequently been defined by clinical, histopathologic and genetic observations $[1-3,5]$.

The study of the Family OSU demonstrated that there are multiple phenotypes that have evolved over decades in this lamin A/C mutation. Sinus node and AV node fibrosis and dysfunction lead to sinus bradycardia and mild AV conduction abnormalities at early stages of the disease, while high-grade AV block requiring permanent pacemaker implantation occurs several years later. Atrial myopathy due to interstitial fibrosis results in atrial enlargement and dysfunction leading to the development of atrial arrhythmias in the third and fourth decades of life [4]. Left ventricular fibrosis produces left ventricular dysfunction and heart failure, which usually occurs in the fifth to seventh decades of life. Sudden cardiac death most likely due to ventricular tachycardia/fibrillation may occur at any stage of the disease, but usually occurs at advanced stages $[1,3,4]$.

Although the cardiovascular disorders associated with lamin A/C mutation are broad, emphasis has been placed on dilated cardiomyopathy as the primary association. The Family OSU study presents evidence of sequential hierarchical progression through multiple cardiac phenotypes on a decade-to-decade basis. In this setting, each phenotype may be mistakenly considered as a specific single diagnosis by physicians working without a pedigree or long-term follow-up. Genetic analysis, however, confirms the LMNA mutation (fig. 6).

From the study it is obvious that while the genetic abnormality is the same, the phenotypic expression of the disease (that is, age of initial presentation, progression and death) were different in the various families. Of interest, the phenotype and progression of disease in the same immediate family members were similar. The fact that the phenotypic expression tends to be similar within the same family members suggests that genetic modifiers and not only environmental factors play an important role. Recognition of these abnormalities and the family history combined with genetic analysis form the basis for clinical decisions, especially for ICD implantation and future therapeutic interventions.

Although lamin A/C is present in all differentiated cells of the body, the gene abnormality in the Family OSU involves mostly the heart. The mechanisms responsible for this phenotypic expression are not completely understood. Abnormal function of chromosome 6 protein which is expressed mostly in the heart and interacts with lamin A/C may at least partially explain this phenomenon or at least provide insight to the possibility of abnormal protein-to-protein interactions as a potential mechanism on why certain laminopathies affect only certain organs [12].

Although the role of the basic scientist in heritable cardiac disorders is of great significance, the present study was initiated by the clinical investigator. The role of the clinical investigator with long-term careful clinical observations of the individual patient was essential to establish the phenotype at different stages of the disease [1, 3 , 4]. The basic scientist defined the genetic abnormalities $[2,6]$. It was the clinician, however, who defined the different phenotypic expressions in different families, although the genetic abnormality was the same. Thus, patient-oriented research is fundamental. Clinical manifestations of the disease may vary from patient to patient which will be defined by the clinical investigator and in turn help the basic scientist to define genetic modifying factors, but not the other way around. The Family OSU emphasizes the importance of clinical observation in the advancement of biomedical sciences.

The role of the physician and the basic scientist for the study of heritable disorders are equally important but different $[17,18]$. Only the physician-scientist, however, who is in constant contact with the patient understands the complexity of the disease. The physician-scientist with an interest in a particular disease can guide the basic scientist to define molecular mechanisms of that disease and by extension learn important lessons for other diseases [17].

Cardiology 2011;118:179-186

Heritable Cardiac Conduction and 


\section{References}

-1 Graber HL, Unverferth DV, Baker PB, Ryan JM, Baba N, Wooley CF: Evolution of a hereditary cardiac conduction and muscle disorder: a study involving a family with six generations affected. Circulation 1986;74: 21-35.

$\checkmark 2$ Kass S, MacRae C, Graber HL, Sparks EA, McNamara D, Boudoulas H, Basson CT, Baker PB, Cody RJ, Fishman MC, Cox N, Kong A, Wooley CF, Seidman JG, Seidman CE: A gene defect that causes conduction system disease and dilated cardiomyopathy maps to chromosome Ip1-Iq1. Nat Genet 1994;7:546-551.

$\checkmark 3$ Nelson SD, Sparks EA, Graber HL, Boudoulas $\mathrm{H}$, Mehdirad AA, Baker PB, Wooley C: Clinical characteristics of sudden death victims in heritable (chromosome Ip1-Iq1) conduction and myocardial disease. J Am Coll Cardiol 1998;32:1717-1723.

4 Sparks EA, Graber HL, Boudoulas H, Nelson SD, Baker PB, Wooley CF: Atrial myopathy and atrial fibrillation: phenotypes in heritable cardiac conduction and myocardial disease. Eur Heart J 2000;(suppl K):K78-K90.

5 Baker PB, Sparks E, Raman S, Wooley C: Histopathologic substrate for clinical manifestations and imaging in heritable cardiac conduction system and myocardial disease. 26th Congress of the International Association of Pathologists. 2006, Toronto, Canada.
6 Fatkin D, MacRae C, Sasaki T, Wolff MR, Porcu M, Frenneauz M, Atherton J, Vidallet HJ, Spudich S, De Girolami U, Seidman JG, Seidman CE: Missense mutations in the rod domain of the lamin A/C gene as causes of dilated cardiomyopathy and conductionsystem disease. N Eng J Med 1999;341:17151724.

7 Stuurman N, Heins S, Aebi U: Nuclear lamins: their structure, assembly, and interactions. J Structural Biology 1998;122:42-66.

-8 Bonne G, DiBarletta MR, Varnous S, Becane Greenberg CR, Gary F, Urtizberea JA, Duboc D, Faredau M, Toniolo D, Schwartz K: Mutations in the gene encoding lamin A/C cause autosomal dominant Emery-Dreifuss muscular dystrophy. Nat Genet 1999;21:285288.

9 Brodsky G, Muntoni F, Miocic S, Sinagra G, Sewry C, Mestroni L: Lamin A/C gene mutation associated with dilated cardiomyopathy with variable skeletal muscle involvement. Circulation 2000;102:473-476.

$>10$ Hutchinson CJ, Alvarez-Reyes M, Vaughan OA: Lamins in disease. Why do ubiquitously expressed nuclear envelope proteins give rise to tissue-specific disease phenotypes? J Cell Science 2001;114:9-19.

$>11$ Nigg EA: Assembly and cell cycle dynamics of the nuclear lamina. Semin Cell Biol 1992; 3:245-253.

12 Boudoulas KD, Burgon PG, Wang L, Conner DA, Zon L, Pizard A, Shin JT, MacRae CA, Seidman JG, Seidman CE: C6P: A novel cardiac protein that interacts with lamin A/C. Circulation 2005;112(suppl):186.
13 Grines CL, Bashore TM, Boudoulas H, Olson S, Shafer P, Wooley CF: Functional abnormalities in isolated left bundle branch block: the effect of interventricular asynchrony. Circulation 1989;39:845-853.

14 Raman SV, Sparks EA, Baker PB, McCarthy B, Wooley CF: Midmyocardial fibrosis by cardiac magnetic resonance in patients with lamin $\mathrm{A} / \mathrm{C}$ cardiomyopathy: possible substrate for diastolic dysfunction. J Cardiov Magn Res 2007;9:907-913.

15 Raman SV, Ng VY, Neff MA, Sayar S, Sparks EA, Nelson SO, Ferketich AK, Wooley CF: Volumetric cine CMR to quantify atrial structure and function in patient with atrial dysthrythmias. J Cardiol Man Res 2005;7: 539-543.

16 Wu W, Muchir A, Shan Y, Bonne G, Worman HJ: Mitogen-activated protein kinase inhibitors improve heart function and prevent fibrosis in cardiomyopathy cased by mutation in Lamin A/C. Circulation 2011;123:53-61.

17 Brown MS: The making of a physician-scientist; in Cook Grosman D, Valtin H (eds): Great Issues for Medicine in the Twenty First Century. New York, Ann NY Academy of Science, 2000, pp 247-256.

18 Levin LA, Danech-Meyer HV: Lost in translation. Bumps in the road between bench and bedside. JAMA 2010;303:1533-1534. 